\title{
On crack path stability in a layered material
}

\author{
J. Gunnars, P. Ståhle, T. C. Wang
}

\begin{abstract}
Crack paths in an elastic layer on top of a substrate are considered. Crack growth is initiated from an edge crack in the layer. The plane of the initially straight crack forms an angle to the free surface. The load consists of a pair of forces applied at the crack mouth and parallel to the interface. Crack paths are calculated using a boundary element method. Crack growth is assumed to proceed along a path for which the mode II stress intensity factor vanishes. The inclination and the length of the initial crack are varied. The effect of two different substrates on the crack path evolution is demonstrated. A crack path initially leading perpendicularly to the interface is shown to be directionally unstable for a rigid substrate. Irrespective of its initial angle, the crack does not reach the interface, but reaches the free surface if the layer is infinitely long. At finite layer length the crack reaches the upper free surface if the initial crack inclination to the surface is small enough. For an inextendable flexible substrate, on the other hand, the crack reaches the interface if its initial inclination is large enough. For the flexible substrate an unstable path parallel with the sides of an infinitely long layer is identified. The results are compared with experimental results and discussed in view of characterisation of directionally unstable crack paths. The energy release rate for an inclined edge crack is determined analytically.
\end{abstract}

\section{1}

\section{Introduction}

Protective coatings are often used to improve mechanical, thermal and chemical performance of devices. As an example, ceramic coatings, with their high hardness and high-temperature corrosion resistance, are used to protect tough materials from excessive wear. Under some conditions chipping of the coating, without delamination, is observed. Thus, the mechanisms for formation of frag-

Communicated by P. E. O'Donoghue, M. D. Gilchrist,

K. B. Broberg, 6 January 1997

J. Gunnars, P. Ståhle

Division of Solid Mechanics, Luleå University of Technology, Sweden

T. C. Wang

Institute of Mechanics, Chinese Academy of Science, China

Correspondence to: J. Gunnars

The authors are indebted to Dr W. L. Zang for providing source code of the boundary element program RESULT-O. Financial support from TFR is gratefully acknowledged. ments (debris) of brittle coatings are important in the understanding of the wear behaviour.

Experimental evaluation of brittle coatings often involves pressing an indenter into the surface, and observing the ensuring crack pattern, see e.g. Larsson et al. (1996). Surface cracks start growing at the indenter, or at flaws in the film or at the interface when the load reaches a critical level. A crack that grows towards the interface may a) continue straight ahead through the interface, b) continue straight ahead and then kink-off $90^{\circ}$ at the interface causing debonding, and c) follow a curved path that may approach the interface at a slanting angle, may arrest in the coating, or may return to the free surface. A noteworthy aspect is that the adhesive properties of the interface are not primarily involved in case $c$.

Investigation of crack trajectories is also interesting in view of the possibility to deflect and/or arrest a crack before it reaches an interface. A number of investigations have recently been conducted on the mechanics of cracks approaching interfaces. An extensive review is given by Hutchinson and Suo (1991). Some attention has also been devoted to cracks at interfaces when one or both of the materials can deform plastically, e.g. Ståhle and Shih (1992) and Sugimura et al. (1995). In nearly all cases the cracks were assumed to be straight and perpendicular to the interface and in some cases crack arrest was predicted. However, in a non-homogeneous remote stress field (or in an inhomogenous material) an originally straight crack will grow along a curved path. Prediction of such paths can only be accomplished by numerical means. Using finite element methods results in the need of extensive remeshing of the element discretisation as the crack grows. By using boundary element methods the time consuming rediscretisation is reduced. Some recent investigations utilising boundary element methods for crack path prediction are; Bower and Fleck (1994), investigating growth of edge cracks formed in an infinite half-plane at a sliding contact, and Selcuk et al. (1994), studying fracture trajectories from cracks close to the interface in bimaterial specimens applied to tensile loading perpendicular to the interface.

In an experimental study by Gunnars et al. (1994) cracks growing in a polycarbonate layer bounded to a steel layer were found to approach or to avoid the interface between the two materials depending on material properties and geometry. An initial edge crack, closely perpendicular to the interface was introduced, and mode I loading was applied. The general observation was that the crack did not follow a straight path, but deflected gradually. Finally the crack appeared to run parallel with the 


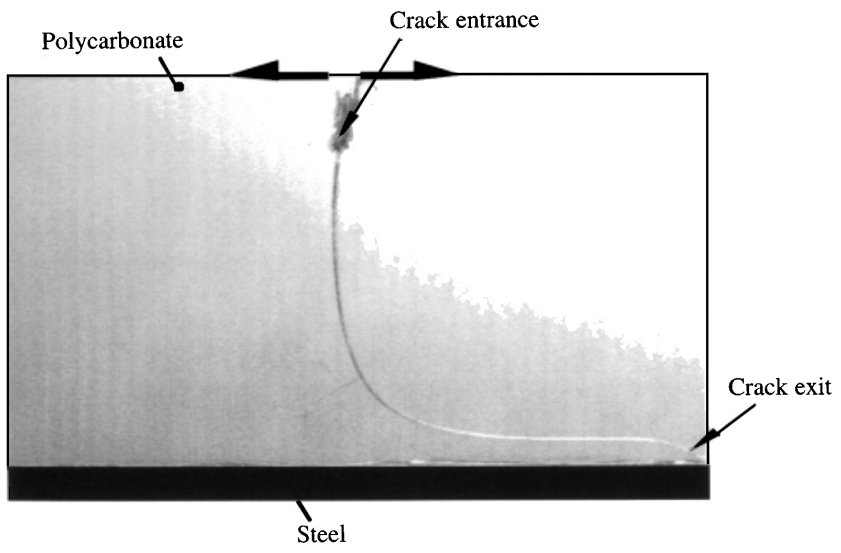

Fig. 1. Crack path obtained at experiments with polycarbonatesteel bimaterial specimens

interface (see Fig. 1). Only for very thin steel layers did the cracks run straight ahead along the original crack plane, down to the interface. An interesting question is whether the path of a crack perpendicular to the interface is stable. Another question is whether a stable path parallel with the interface exists for large specimens. The experiments indicated that the finite dimension of the specimens may have an influence on the results.

The present paper investigates the growth of an edge crack into an elastic layer on top of a substrate, assumed to be much stiffer than the layer. A boundary element method is used for calculation of the crack paths. The crack is assumed to follow a path such that the mode II stress intensity factor vanishes at the crack tip. The inclination and the length of the initial crack are varied. The influence of different geometries (layer and substrate thicknesses and length) on the resulting pattern of fracture is studied.

\section{2}

\section{The problem}

A crack approaching the interface in a bimaterial strip subjected to a surface contact is considered. The twodimensional model illustrated in Fig. 2 is analysed. Only the compliant layer containing the surface crack is shown. The material is homogenous, isotropic and linear elastic. The (ideally brittle) layer containing the crack has a modulus of elasticity $E$ and Poisson's ratio $v$. The height of

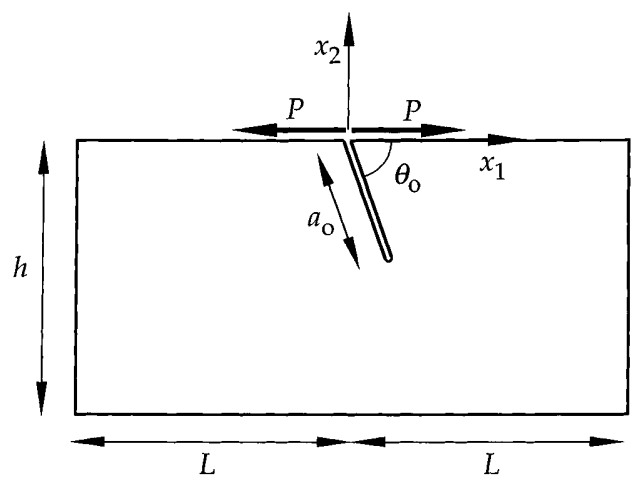

Fig. 2. Geometry and loading for the initial edge crack problem the layer is $h$ and the length of the layer is $2 L$. The layers are perfectly bonded at the interface $x_{2}=-h$. Plane strain conditions are assumed. The edge crack has an initial length $a_{0}$. The initial crack is included an angle, $\theta_{0}$, to the $x_{1}$-axis and meets the surface at $x_{1}=x_{2}=0$. At $x_{1}=x_{2}=0$ two forces $P$ parallel with the $x_{1}$-axis are applied to opposed crack surfaces so that they tends to open the crack. The loading is chosen as a simple model of the opening loading of a surface crack behind a passing contact with friction, or can represent the forces at a pit in a contact between rough surfaces.

All boundaries except the interface are traction free surfaces, that is $\sigma_{22}=\sigma_{12}=0$ at $x_{2}=0$ and $0<\left|x_{1}\right| \leq L$, and $\sigma_{11}=\sigma_{12}=0$ at $\left|x_{1}\right|=L$ and $-h<x_{2}<0$. The substrate is assumed to be much stiffer than the layer containing the crack, and two cases are considered. The first case is a very thick substrate layer, modelled by rigid boundary conditions at the interface. The second case is a very thin substrate layer (as compared to the compliant layer). In this case the bending stiffness is assumed to be negligible (fully flexible), i.e. the normal traction vanishes at the interface. However, the longitudinal stiffness is assumed to be high, thus, making the interface inextendable. The cases correspond to extreme cases of the experimental study by Gunnars et al. (1994) on polycarbonate-steel specimens. Thus, the boundary conditions at the interface, $x_{2}=-h$ and $\left|x_{1}\right| \leq L$, are

case 1: $\quad u_{1}=u_{2}=0, \quad$ (rigid substrate)

or

case 2: $\quad \sigma_{22}=0 ; u_{1}=0$

$$
\text { (inextendable flexible substrate ) }
$$

The load is assumed to be high enough to cause fracture and the resulting pattern of fracture is analysed. The crack is assumed to grow quasi-statically. The crack faces are assumed not to make contact with each other.

\section{3}

\section{Experiments}

In Gunnars et al. (1994) crack paths were studied experimentally in a bimaterial system, see Fig. 1. Specimens were made of polycarbonate and steel, and the propagation of an edge crack in the polycarbonate was studied.

The dimensions of the test specimens were: height $h=50 \mathrm{~mm}$, height of the substrate layer $h_{b}=0.3$ to $200 \mathrm{~mm}$ and length $L=h$ or $2 h$. The materials were bonded by an epoxy adhesive. The elastic modulus and Poisson's ratio for the polycarbonate and steel were $E=2.5 \mathrm{GPa}, v=0.38$, $E_{b}=205 \mathrm{GPa}$ and $v_{b}=0.31$, respectively. An initial crack perpendicular to the interface, with length $8.75 \mathrm{~mm}$, was made. Grooves were made at the notch mouth to attach the loading grips. The load was applied perpendicularly to the crack at the notch mouth, on the free upper surface of the polycarbonate. The specimen was carefully aligned to assure pure mode I loading of the initial crack (checked by use of a polariscope).

The crack was made to grow in the polycarbonate under chemical assistance by adding a drop of acetone to the crack tip. The crack propagated with a speed less than $1 \mathrm{~m} / \mathrm{s}$. The fracture surface had a brittle appearance. 
Normally polycarbonate is a very ductile material, but plastic yielding was not observed. Thus, by use of solvent assisted crack growth polycarbonate can be utilised for studying elastic crack paths for low crack speeds.

Crack paths where obtained for the different ratios $h / h_{b}$. The crack seemed to grow straight towards the interface until it suddenly curved off to follow a path close to the interface, see Fig. 1. In the case of a very thin steel layer the crack path continued straight to the interface. By comparing photoelastic and finite element results, the crack tip was found to be embedded in an elastic field. The finite element analysis showed that the mode II stress intensity factor was less than $2 \%$ of the mode I stress intensity factor along experimentally obtained paths. In this paper some of the experimental crack paths will be compared with numerically predicted paths.

\section{4}

\section{Crack path criteria}

Several hypothesis may be adopted for the prediction of the direction of crack growth at complex stress states. Let the mixed mode stress field at the crack tip be described by the opening mode (mode I) stress intensity factor $K_{\mathrm{I}}$ and the shearing mode (mode II) stress intensity factor $K_{\mathrm{II}}$, and introduce the ratio $m=K_{\mathrm{II}} / K_{\mathrm{I}}$. Two commonly used criteria for fracture of brittle materials are: crack extension in the plane perpendicular to the maximum tensile stress, and propagation in the direction of maximum strain energy release rate, both proposed by Erdogan and Sih (1963). From experimental facts and based on that the micro-separation mechanisms in opening and sliding are fundamentally different, Broberg (1987) argues that at mixed mode I + II fracture the crack grows in a direction that maximises $K_{\mathrm{I}}$ or $K_{\mathrm{II}}$, rather than in a direction that maximise some functional of $K_{\mathrm{I}}$ and $K_{\mathrm{II}}$. Further, experimental observations indicate that mode I crack growth is usually preferred before mode II. Exceptional behaviour may occur when the ratio between the critical stress intensity factor for modes II and I is small or when compressive stresses are present. See Broberg (1987), for a detailed discussion of directional criteria. Thus, we assume that the crack grows in the direction that maximises $K_{\mathrm{I}}$ (Kalthoff 1973). For a smooth path this criterion coincides with vanishing $K_{\text {II }}$ at the local crack tip, i.e. pure opening mode.

However, Bergkvist and Guex (1979) show that the predicted crack path to a large extent is independent of the choice among most of the generally used directional criteria, except for the initial kink. For small deviations from pure mode $\mathrm{I}(m \ll 1)$ the following relation can be used for prediction of the direction of crack extension

$\Delta \theta=-2 m+\mathrm{O}\left(m^{2}\right)$

where $\Delta \theta$ is the angle between the previous crack plane and the new direction of crack extension. Thus, the crack extends by a kink at initiation of crack growth from a mixed-mode loaded virgin crack. The continued crack growth is assumed to follow a smoothly curved path (small kink angles) for which $m$ vanishes at all times.

In the discussion of crack path selection the concept of directional stability is often used. The importance of the definition of directional stability of a straight path is noted by Melin (1983). Introducing an infinitesimal disturbance at the tip of a straight mode I crack under symmetric loading in an infinite body, she studies the subsequent growth. The straight crack is defined as directionally stable if the crack direction eventually approaches the original one, and unstable if the crack direction deviates more and more from the original one. An alternative more severe definition is to require that the crack will approach the plane of the original straight crack. With the first definition that a directionally stable path eventually will approach a direction parallel to the original crack, Melin $(1983,1992)$ arrive at the result that the straight path is unstable for $\sigma_{x}^{\infty} / \sigma_{y}^{\infty}>1$, in the case of a crack with remote stresses $\sigma_{x}^{\infty}$ and $\sigma_{y}^{\infty}$, the latter perpendicular to original crack. Discussion on the characterisation of crack paths is also found in Melin (1991).

\section{5}

\section{Numerical method}

Crack paths were simulated by use of a boundary element method (BEM). The method is based on two coupled integral equations. On the outer boundary tractions and displacements are treated as unknowns (standard BEM), and along the crack line dislocation densities are the unknowns, see Zang and Gudmundson (1989). This modified formulation performs excellently even when kinks are present along the crack path. In addition only one of the crack surfaces has to be modelled. The formulation employs integral kernels based on the fundamental solution for a infinite half-plane.

The crack is modelled by piece-wise straight segments, each discretised into a number of elements. A linear isoparametric interpolation is employed for the dislocation densities. A two-node singular element is used adjacent to the crack tip. The integral equations are solved by a collocation method.

In the calculations the initial edge crack consists of a short crack mouth segment and a longer segment containing the crack tip. The short segment meets the free surface at $x_{1}=0$, and has a length of $a_{0} / 35$. This segment is perpendicular to the free surface and is loaded with a traction normal to the segment, see Fig. 3. The perpendicular segment is necessary for application of the loading in question, since crack lines are assumed to be symmetrically loaded in the boundary integral formulation. The angle between the longer segment and the free surface is $\theta_{0}$. Since the kernel functions satisfy the free half space condition, there is no need to discretise the upper free surface.

Crack growth is simulated by repeated calculations of $K_{\mathrm{I}}$ and $K_{\mathrm{II}}$ (from the dislocation densities at the crack tip node), followed by calculation of $\Delta \theta$ according to (3). After each calculation a new segment is added in the predicted direction. All segments have length $\Delta a=h / 100$ and are straight. Within each segment 6 elements are used, except for the segments containing the crack tip. In this segment 24 elements are used. The ratio $K_{\mathrm{II}} / K_{\mathrm{I}}$ is generally of the order of $10^{-2}$, and is never larger than $10^{-1}$, along the computed paths. The ratio is highest at the first kink (especially for cases of a large initial mode II component, i.e. at small $\theta_{0}$ ) and where the path curves rapidly. In these 


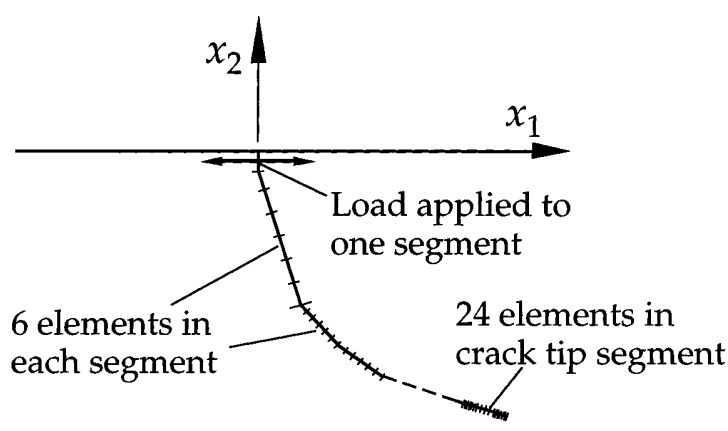

Fig. 3. Boundary element discretisation

cases the path is sensitive to the length of the crack extension, $\Delta a$, and to the discretisation. Calculations proceed until the crack meets a free surface or the interface.

The accuracy was verified against an analytically solved problem for an inclined edge crack, see appendix. The results indicated that for a typical discretisation the errors of the stress intensity factors are less then $1 \%$.

\section{6}

\section{Results}

The paths obtained in the experiments described in Sect. 3 showed some scatter. A sensitivity to small deviations from the desired initial crack inclination $\theta_{0}=90^{\circ}$ was noted. Thus, for example to analyse this experiment, crack paths computed for different initial angles $\theta_{0}$ are of interest. The initial angle is varied from $90^{\circ}$ to $20^{\circ}$. The effect of different initial crack lengths is also studied and three initial lengths $a_{0}=0.175 h, 0.088 h, 0.044 h$ are used. The influence of a finite geometry of the layer is studied by performing all calculations for $L / h=1, L / h=2$ and $L / h=10$, the latter assumed to be a satisfactory approximation for the idealised geometry $L / h \rightarrow \infty$. The calculations were performed for case 1 (rigid substrate) and case 2 (flexible substrate), see (1) and (2).

\section{1}

\section{Influence of initial angle and finite geometry}

Figure 4 shows crack propagation paths as functions of initial angle $\theta_{0}$ for three different lengths $L / h$ for case 1 . The initial crack length $a_{0}=0.175 h$ is kept fixed. Only half of the body is shown. For the infinite layer in Fig. 4a $(L / h \rightarrow \infty)$ the paths return towards the surface for all angles, except exactly $90^{\circ}$. For $\theta_{0}=89.99^{\circ}$ the crack is still deflected at a significant distance from the interface. Thus, the $90^{\circ}$ path is very sensitive to any deviation from the straight path.

By comparing Figs. $4 \mathrm{a}(L / h \rightarrow \infty)$ and $4 \mathrm{~b}(L / h=2)$ the effect of finite dimension of the body can be studied. It is seen that the paths are markedly influenced by the finite geometry after a short extension (about $0.5 h$ ) despite the relatively long distance $(1.5 h)$ to the traction free boundary at $x_{1}=L$. This free boundary effect indicates the effect of a neighbouring crack or hole in a coating on a stiff substrate.

The paths for large angles $\theta_{0}$ in Figs. $4 \mathrm{~b}$ and $4 \mathrm{c}, \mathrm{cp}$.

Fig. 1, (paths approximately parallel with the interface) could possibly be misinterpreted as indications of paths at steady-state depths (see Ståhle et al. 1995). However, the results show that the appearance of these paths is an effect of the traction free boundary.

For sufficiently small initial angles $\theta_{0}$ the cracks return to the free surface at $x_{2}=0$ independently of the geometry. For the finite geometries a characteristic initial angle divides the paths into cracks returning to the boundary at $x_{2}=0$ and those reaching the boundary at $x_{1}=L$. For the geometry $L / h=2$ (Fig. $4 \mathrm{~b}$ ) the angle is approximately $76^{\circ}$ and for $L / h=1$ (Fig. $4 \mathrm{c}$ ) around $30^{\circ}$.

Figure 5 shows crack paths as functions of initial angle $\theta_{0}$ for three different lengths $L / h$ for the flexible substrate (case 2). The initial crack length is constant $a_{0}=0.175 h$. For this interface boundary condition the crack paths show a different pattern. The paths are less influenced by the finite geometry than in case 1 . For all three layer lengths cracks at a sufficiently large initial angle $\theta_{0}$ will terminate at the interface. The smallest angle for this is around $35^{\circ}$ for $L / h=1,30^{\circ}$ for $L / h=2$ and $29.7^{\circ}$ for
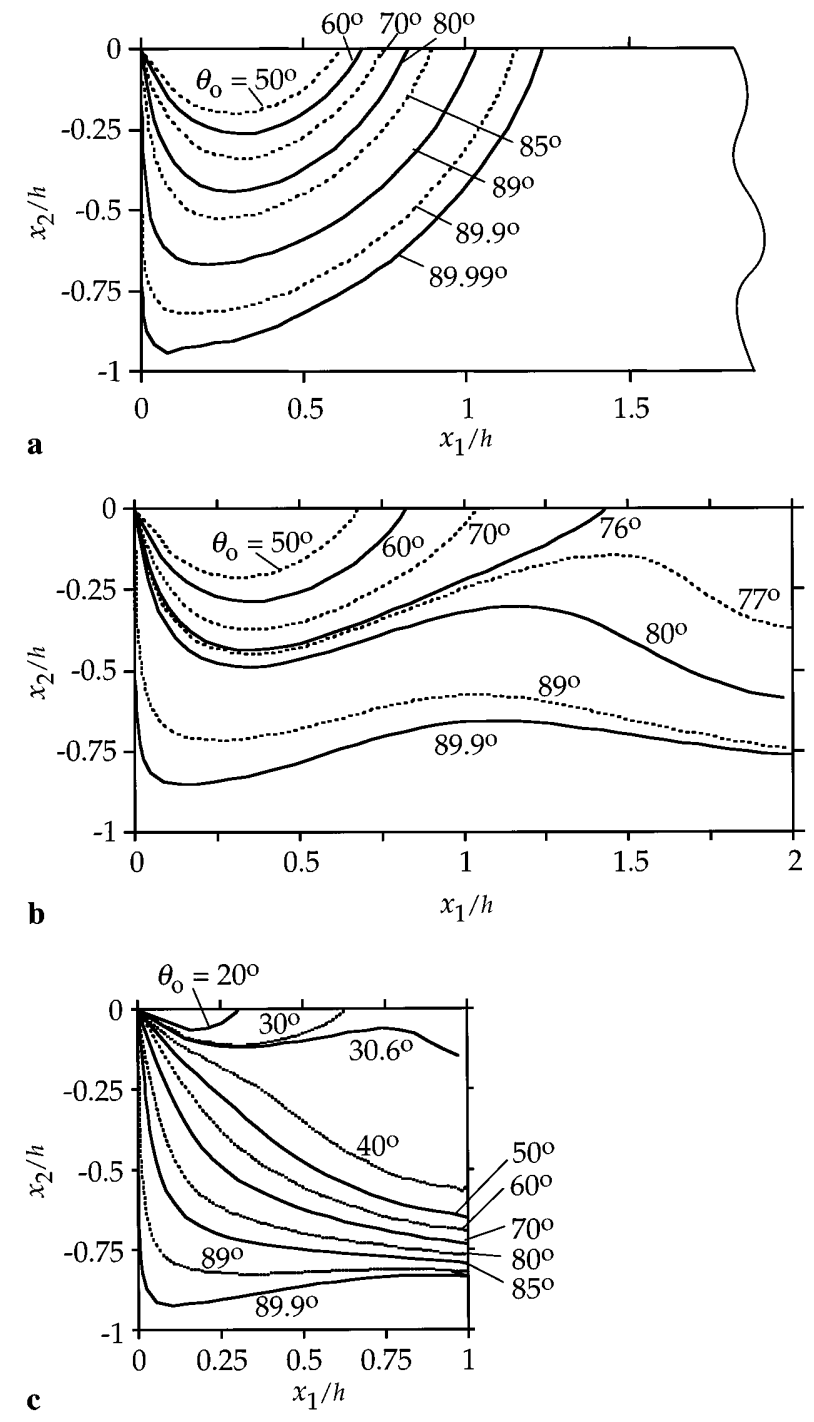

Fig. 4a-c. Crack paths for different $\theta_{0}$ for a rigid substrate (case 1). The initial crack length is $a_{0}=0.175 h$ and the layer length is a $L / h \rightarrow \infty, \mathbf{b} L / h=2$ and c $L / h=1$ 

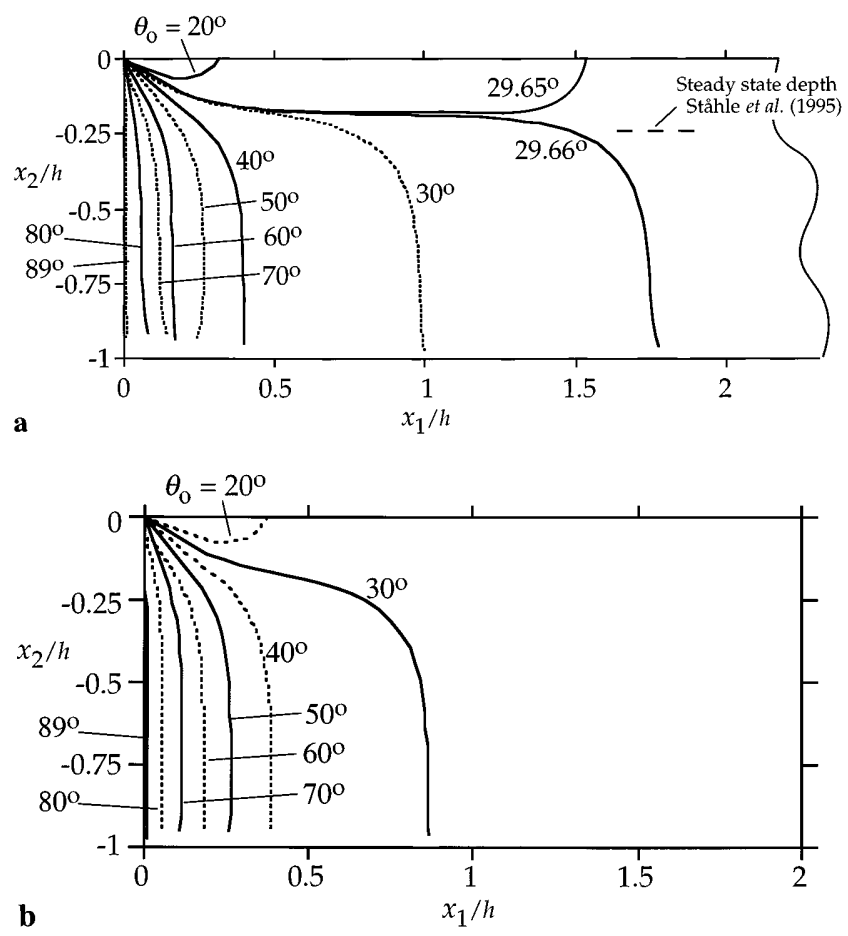

b

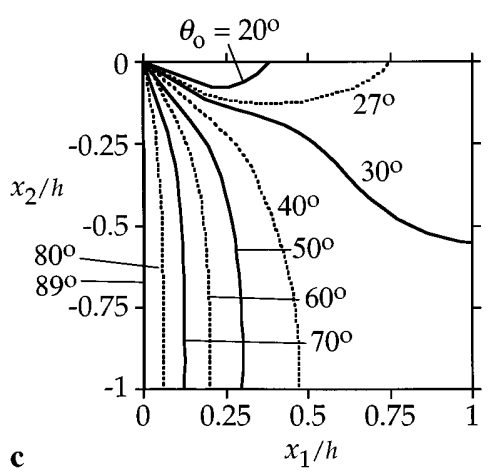

Fig. 5a-c. Crack paths for different $\theta_{0}$ for an inextendable flexible substrate (case 2). The initial crack length is $a_{0}=0.175 h$ and the layer length is a $L / h \rightarrow \infty, \mathbf{b} L / h=2$ and c $L / h=1$

$L / h \rightarrow \infty$. When the crack is very close to the interface the path is influenced by the discretisation of the interface boundary, and thus the simulations were interrupted. To study this final path mesh refinements are needed. At the upper free surface such problems are avoided since no elements are needed at this boundary.

Also in the case 2 cracks at a sufficiently small initial angle $\theta_{0}$ will return to the upper free surface. The limiting angle was found to be approximately $28^{\circ}$ for $L / h=1,29^{\circ}$ for $L / h=2$ and $29.7^{\circ}$ for $L / h \rightarrow \infty$. The constant depth followed for the limit angle in the case $L / h \rightarrow \infty$ agrees fairly well with the steady-state crack depth (indicated in Fig. 5a) predicted by Ståhle et al. (1995). The steady-state path also seems unstable, as predicted by a simple beam model in the same paper.

One observation is that any point in the layer could possibly be reached by selecting the proper initial angle, assuming that no disturbances are introduced along the path. A summary of limiting initial angles $\theta_{0}$ that lead the crack to return to the free upper surface or to the interface are given in Table 1.

For the loading used $K_{\mathrm{I}}$ is positive along all crack paths. Crack face contact behind the tip could possibly occur for some of the paths returning towards the upper free surface, and thus influence the stress intensity ratio $m$.

\section{2}

\section{Different initial crack lengths}

Figure 6 shows the crack path pattern for different initial crack lengths $a_{0}$. The boundary condition at case 2 and the geometry $L / h \rightarrow \infty$ is used. The initial crack angles studied are $\theta_{0}=20^{\circ}, 50^{\circ}$ and $80^{\circ}$. For the two largest angles virtually no effect of the initial length is observed, since the direction of the subsequent growth is virtually the same for all three lengths. For the smallest angle $\theta_{0}=20^{\circ}$, a larger initial crack length seems to lead the crack in a steeper slope to the free surface. Here the starting point for the crack growth is critical, and the paths for different initial lengths can cross over each other.

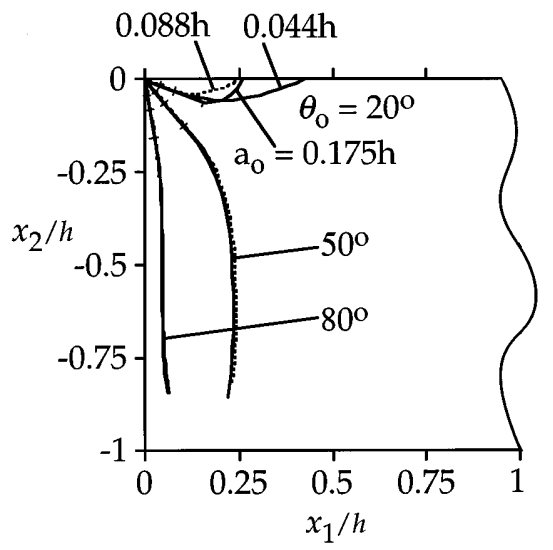

Fig. 6. Crack paths for three different initial crack lengths $a_{0}$ for an inextendable flexible substrate (case 2). Paths are shown for initial crack angles, $\theta_{0}=20^{\circ}, 50^{\circ}$ and $80^{\circ}$
Table 1. Extreme initial edge crack angles $\theta_{0}$ that leads the crack to return to the free upper surface or to the interface, $\left(a_{0}=0.175 h\right)$. In case 2 with $L / h \rightarrow \infty$ the crack follows a steady-state path

\begin{tabular}{lccl}
\hline Case & $l / h$ & $\begin{array}{l}\text { max } \theta_{0} \text { leading } \\
\text { to the upper surface }\end{array}$ & $\begin{array}{l}\text { min } \theta_{0} \text { leading to } \\
\text { the interface }\end{array}$ \\
\hline 1. Rigid substrate & 1 & $30^{\circ}$ & $90^{\circ}$ \\
& 2 & $76^{\circ}$ & $90^{\circ}$ \\
2. Flexible substrate & $\infty$ & $\rightarrow 90^{\circ}$ & $90^{\circ}$ \\
& 1 & $28^{\circ}$ & $35^{\circ}$ \\
& 2 & $29^{\circ}$ & $30^{\circ}$ \\
& $\rightarrow \infty$ & $29.7^{\circ(-)}$ & $29.7^{\circ(+)}$ \\
\hline
\end{tabular}


7

\section{Discussion}

Generalising the definition of directional instability of a straight path given by Melin (1983) leads to the following criterion for a curved path. A given curved path is called directionally unstable if a small deviation $\Delta d_{0}$ at the arclength $s_{0}$ along this path (see Fig. 7a) leads to a deviation $\Delta d$ after the arc-length $s$, such that

$\frac{\Delta d}{s} \gg \frac{\Delta d_{0}}{s_{0}}$

when $s / s_{0}$ is sufficiently larger. Due to the finite extent of the body considered, the limit of what is very large would be necessary to determine. The instability criterion (4) is however certainly fulfilled for paths where $\Delta d_{0} \rightarrow 0$ gives a finite $\Delta d$. By this choice two paths are identified as directionally unstable paths, see Fig. $7 \mathrm{~b}$ and $7 \mathrm{c}$. For case 1 (rigid substrate) and all lengths of the layer the straight path for the initial angle $\theta_{0}=90^{\circ}$ is unstable. The path is directionally unstable since an infinitesimal deviation from $\theta_{0}=90^{\circ}$ will lead the crack in a path eventually ending on the free surface. The second path is the steady-state path reached for an initial angle $\theta_{0} \approx 29.7^{\circ}$ in the infinite strip at case 2 (flexible substrate). This path approaches a direction parallel to the free surface. The path is unstable since even the smallest disturbance will lead the crack to either the bimaterial interface or to the upper free surface.

Thus, most of the calculated paths are characterised as directionally stable, although a small perturbation of the initial angle in most cases leads to a gradually growing distance $\Delta d$ from the original path. However, some more markedly directionally stable paths are identified in regions where $\Delta d$ is not increasing as the crack grows. For the rigid substrate and $x_{1} / h>0.5$ (Fig. $4 \mathrm{a}$, when the cracks are directed towards the upper surface) disturbances will lead to paths paralleling each other. In Fig. 5b paths for initial angles $\theta_{0}>30^{\circ}$ and in the region $x_{2} / h<-0.5$ will also parallel each other after a perturbation. The position where the crack meets the boundary can only be predicted by knowledge of the perturbation, whereas the direction is constant. No paths with the characteristic that the original path is approached after a perturbation (strongly stable paths), that is paths where $\Delta d$ decreases rapidly, were found.

For a finite body, a prediction of the behaviour of a crack solely from the directional stability characterisation of the crack normally provides little information. In general the deviation from a trajectory due to a disturbance is scaling with the size of the disturbance. Only for a strongly stable path the deviation at the boundary is small for a large disturbance if the body is large enough.

Figure 8 shows a comparison between two paths obtained in the experiment described in section 3 and simulated paths. The specimen length is $L=h$, but only half the specimen width is shown. Note that no disturbances are introduced along the numerical paths but only the effect of an initial (angular) disturbance (corresponding to a misalignment) is studied. However, sensitivity to random disturbances during growth is also anticipated from the crack path patterns. Figure 8a is a digitised photograph of a crack path in polycarbonate on a thick $\left(h_{b}=2 h\right)$ steel substrate which should correspond to case 1 (rigid substrate). The numerical paths for $\theta_{0}=89.5^{\circ}, 88^{\circ}$ and $85^{\circ}$ are included. The comparison shows that the alignment at the experiment is very important for the resulting path. This is not a surprise considering the instability of the $\theta_{0}=90^{\circ}$ path. Figure $8 \mathrm{~b}$ is the result for polycarbonate on a thin $\left(h_{b}=0.0075 h\right)$ steel substrate which is supposed to correspond to case 2 (flexible substrate). Here the paths for $\theta_{0}=89.5^{\circ}, 80^{\circ}$ and $70^{\circ}$ are included. Figure $8 \mathrm{~b}$ shows that the alignment is less decisive in this case. However, for this boundary condition loading misalignments due to rotation of the test specimen was more easily introduced during fracture. The path seems rather straight, although large perturbations are introduced.

\section{8}

\section{Conclusions}

Propagation paths of cracks in an elastic layer on top of a substrate are studied. The initial crack is an edge crack

a

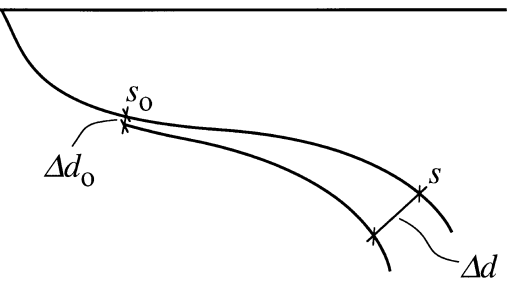

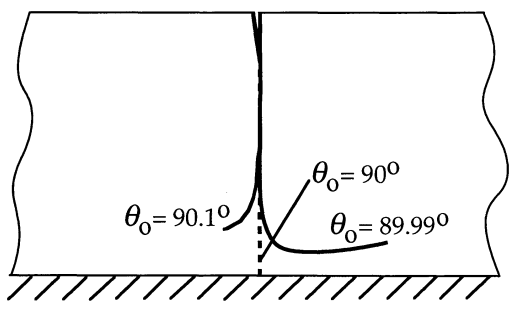

$\mathbf{b}$

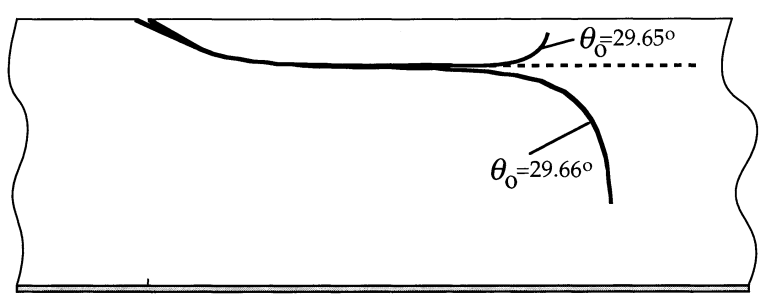

c

Fig. 7. a A curved crack path and the path after introduction of a disturbance. Two unstable paths: $\mathbf{b}$ the $\theta_{0}=90^{\circ}$ path for the rigid substrate, and $\mathrm{c}$ the $\theta_{0} \approx 29.7^{\circ}$ path for the inextendable flexible substrate $(L / h \rightarrow \infty)$ 


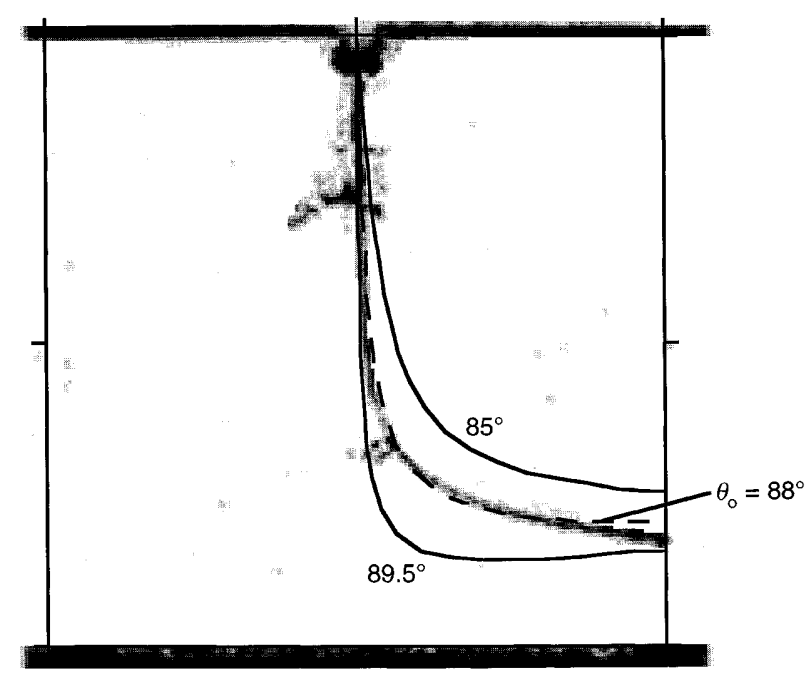

a

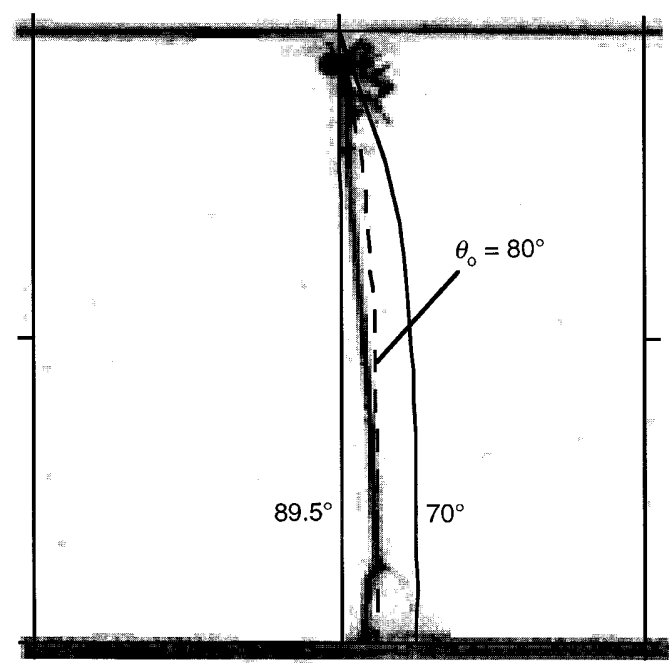

b

Fig. 8a,b. Comparison of experimentally obtained crack paths (Gunnars et al., 1994) with numerically predicted paths. In a numerical paths for case 1 (rigid substrate) are shown for $\theta_{0}=89.5^{\circ}, 88^{\circ}$ and $85^{\circ}$, and in $\mathbf{b}$ paths for case 2 (inextendable flexible substrate) are shown for $\theta_{0}=89.5^{\circ}, 80^{\circ}$ and $70^{\circ}$

Table A1. Numerically and analytically obtained normalised stress intensity factors and energy release rates for edge cracks at different angles $\theta_{0}$ to the surface

\begin{tabular}{|c|c|c|c|c|}
\hline$\theta_{0}$ & $\frac{K_{\mathrm{I}} \sqrt{a_{0}}}{P}$ & $\frac{K_{\mathrm{II}} \sqrt{a_{0}}}{P}$ & $\frac{G E a_{0}}{\left(1-v^{2}\right) P^{2}}$ & $\frac{G E a_{0}}{\left(1-v^{2}\right) P^{2}}$ eq. (A1) \\
\hline $90^{\circ}$ & 1.458 & 0.0000 & 2.125 & 2.141 \\
\hline $60^{\circ}$ & 1.565 & -0.0593 & 2.453 & 2.467 \\
\hline $30^{\circ}$ & 2.035 & -0.1605 & 4.167 & 4.212 \\
\hline $15^{\circ}$ & 2.804 & -0.3098 & 7.956 & 7.985 \\
\hline $5^{\circ}$ & 4.818 & -0.6871 & 23.69 & 23.24 \\
\hline
\end{tabular}

inclined to the free surface and loaded with a pair of forces, parallel to the interface, applied to the crack mouth. The loading serves for example as a simple model of the opening loading of a surface crack behind a passing contact with friction. Crack paths are calculated using a boundary element method, assuming that the crack fol- lows a path such that the mode II stress intensity factor vanishes.

Initial cracks with a small inclination to the free surface return to this surface irrespective of the properties of the substrate. Cracks with a large inclination to the free surface return to the surface in case of a rigid substrate and approach the bimaterial interface for an inextendable flexible substrate. Thus, for a compliant layer on a stiff substrate the resulting fractures are confined to the layer, i.e. substrate exposure is prevented. A very thin substrate on the other hand, tends to attract cracks.

The crack paths are markedly influenced by a finite length of the layer for the rigid substrate, and insensitive to layer length for the inextendable flexible substrate. Even a free surface at a distance $L=2 h$ from the initial crack attracts initial cracks with large inclination for the rigid substrate. For example, this effect indicates the process of successive widening of a pit in a brittle coating on a stiff substrate.

The length of the initial crack influences the final shape of fracture if the direction of growth after initiation is rapidly changing (within the distance of the change of initial length). This is the case for the rigid substrate, and for small initial angles for the flexible substrate.

Two paths are identified as directionally unstable. The symmetry crack path leading perpendicularly to the interface is unstable for a rigid substrate. For the flexible substrate a path parallel with the sides (at a constant depth) of an infinitely long layer is unstable.

A discussion on some experimental crack paths points out that for a stiff substrate the finite dimension of a bimaterial specimen strongly influences the resulting paths.

\section{Appendix}

\section{Analytical solution for the energy release rate for an inclined edge crack opened by concentrated forces and verification of numerical accuracy}

In Freund (1978) an approach is presented for solving a class of plane elastic crack problems by use of the $\mathrm{M}$-integral conservation law. The energy release rate, $G$, can be determined without the need to solve the complete boundary value problem. We consider an inclined edge crack in an infinite half plane. The crack is inclined an angle $\theta_{0}$ to the traction free surface and is under the action of two equal and opposite concentrated forces $P$ parallel to the free surface and applied at the corners at the surface (cp. Fig. 2). Applying the integral conservation law one obtains the mixed mode energy release rate

$G=\frac{\left(1-v^{2}\right)}{E}\left(K_{\mathrm{I}}^{2}+K_{\mathrm{II}}^{2}\right)=\frac{\left(1-v^{2}\right) P^{2}}{E a_{0}} f\left(\theta_{0}\right)$,

where

$f\left(\theta_{0}\right)=$

$2 \pi\left(1+2 \theta_{0}\left(\theta_{0}-\pi\right)-\cos 2 \theta_{0}-\left(2 \theta_{0}-\pi\right) \sin 2 \theta_{0}\right)$ $\overline{\left(1-2 \theta_{0}^{2}-\cos 2 \theta_{0}\right)\left(2 \pi^{2}-1+2 \theta_{0}\left(\theta_{0}-2 \pi\right)+\cos 2 \theta_{0}\right)}$ and $a_{0}$ is the crack length, $E$ is the modulus of elasticity and $v$ is the Poisson's ratio. Table A1 shows a comparison 
between this solution, evaluated for five different angles $\theta_{0}=90^{\circ}, 60^{\circ}, 30^{\circ}, 15^{\circ}$ and $5^{\circ}$, and the results from numerical calculations obtained for $a_{0} / h=0.001, L / h=1$, and typical crack line discretisations. A somewhat finer discretistion is needed for the smaller angles $\theta_{0}$. The error of the energy release rate is less than $2 \%$. Note the rapid growth of $K_{\mathrm{I}}$ and $K_{\mathrm{II}}$ as $\theta_{0}$ tends to zero. The sign of $K_{\mathrm{II}}$ is with reference to a local coordinate system at the crack tip with an axis in the direction of the extension of the crack plane and the other perpendicular to this in the counter clock-wise direction.

\section{References}

Bergkvist, H.; Guex, L. (1979): Curved crack propagation. Int. J. Fract. 15, 429-441

Bower, A. F.; Fleck, N. A. (1994): Brittle fracture under a sliding line contact. J. Mech. Phys. Solids 42, 1375-1396

Broberg, K. B. (1987): On crack paths. Eng. Fract. Mech. 28, 663679

Erdogan, F.; Sih, G. C. (1963): On the crack extension in plates under plane loading and transverse shear. J. Basic Eng. 85, 519525

Freund, L. B. (1978): Stress intensity factor calculations based on a conservation integral. Int. J. Solids Struct. 14, 241-250

Gunnars, J.; Delfin, P.; Ståhle, P. (1994): Quasi-static curved crack paths in a bi-material specimen obtained by chemical assisted fracture in polycarbonate. Report form Division of Solid Mechanics, Uppsala Institute of Technology, Uppsala, Sweden
Hutchinson, J. W.; Suo, Z. (1991): Mixed mode cracking in layered materials. Adv. Appl. Mech. 29, 63-191

Kalthoff, J. F. (1973): On the propagation direction of bifurcated cracks. In: Sih, G.C. (ed.): Dynamic Crack Propagation. Noorddhoff International Publishing, Leyden, 449-458

Larsson, M.; Olsson, M.; Hedenqvist, P.; Hogmark, S. (1996): On the mechanisms of coating failure as demonstrated by scratch and indentation testing of TiN coated HSS (to appear in Surf. Coat. Technol.)

Melin, S. (1983): Why do cracks avoid each other? Int. J. Fract. 23, 37-45

Melin, S. (1991): On the directional stability of wedging. Int. J. Fract. 50, 293-300

Melin, S. (1992): Directional stability of an originally straight crack. Int. J. Fract. 53, 121-128

Selcuk, S.; Hurd, D. S.; Crouch, S. L.; Gerberich, W. W. (1994): Prediction of interfacial crack path: a direct boundary integral approach and experimental study. Int. J. Fract. 67, 1-20 Ståhle, P.; Shih, C. F. (1992): Cracking in thin films and substrates. Materials Research Society Symposium Proceedings 239, Boston, 567-572

Ståhle, P.; Gunnars, J.; Delfin, P. (1995): Crack paths in a weak elastic layer covering a beam. Acta Mech. Solida Sinica 8, S. Issue: FEFG'94 Proceeding of Int. Symp. on Fracture and Strength of Solids, 579-583

Sugimura, Y.; Lim, P. G.; Shih, C. F.; Suresh, S. (1995): Fracture normal to a bimaterial interface: effects of plasticity on crack-tip shielding and amplification. Acta Metall. Mat. 43, 1157-1169 Zang, W. L.; Gudmundson, P. (1989): An integral equation method for piece-wise smooth cracks in an elastic half-space. Eng. Fract. Mech. 32, 889-897 